\title{
Trypanosoma (Megatrypanum) lizae n. sp.
}

un trypanosome ayant des formes géantes chez les Microchiroptères

$$
\text { Hipposideros cyclops au Gabon }
$$

\author{
par F. MILTGEN et I. LANDAU \\ Laboratoire de Zoologie (Vers) associé au C.N.R.S., Muséum national d'Histoire naturelle, \\ 43, rue Cuvier, F 75231 Paris Cedex 05.
}

\section{Résumé.}

Trypanosoma (Megatrypanum) lizae $\mathrm{n}$. sp est un trypanosome dont certaines formes géantes peuvent atteindre $1400 \mu \mathrm{m}$; il a été trouvé chez des Microchiroptères Hipposideros cyclops au Gabon. La morphologie singulière et les caractères des petites formes nous amènent à en faire une nouvelle espèce du sous-genre Megatrypanum.

\section{Summary:}

Trypanosoma (Megatrypanum) lizae n. sp. : a trypanosome with giant forms from the Michrochiroptera Hipposideros cyclops, in Gabon.

Trypanosoma (Megatrypanum) lizae n. sp.: trypanosome with giant forms reaching a length of $1400 \mu \mathrm{m}$ from the Microchiroptera Hipposideros cyclops in Gabon. Because of the peculiar morphology of giant forms and the characteristics of small individuals it is placed in a new species within the subgenus Megatrypanum.

\section{Matériel et méthodes}

Au cours d'une mission au Gabon, en août 1977, nous avons capturé dans les environs de Makokou trois Microchiroptères de l'espèce Hipposideros cyclops (Temminck). Des frottis ont été faits à partir du sang cardiaque des Chauves-souris au 
moment de leur autopsie, ainsi que des appositions d'organes : foie, rate, reins et poumons; nous avons par ailleurs fixé des blocs histologiques au Carnoy. Les coupes ont été colorées au Giemsa-colophane.

\section{Résultats}

Les frottis sanguins se sont révélés intéressants à plus d'un titre : en plus d'une nouvelle espèce de Plasmodium (la première rencontrée chez un Microchiroptère (Landau et Chabaud, 1978), d'un nouvel Haemoproteidae (Landau et coll., en préparation), les chauves-souris étaient porteuses de nombreux trypanosomes. Certains sont de taille modeste (de l'ordre de 20 à $45 \mu \mathrm{m}$, voir Pl. III, B à F) et ont une morphologie typique de Megatrypanum (Hoare, 1964), d'autres sont atteints de gigantisme et ont une morphologie tout à fait inhabituelle.

\section{Description}

a) «Petites formes » (Planches III, B à G).

Ce sont des trypanosomes dont les longueurs totales vont de 20 à $45 \mu \mathrm{m}$. Des formes trypomastigotes et sphaeromastigotes ont été observées; aucune forme de multiplication n'a été vue; les tailles de corps s'échelonnent entre $15 \mu \mathrm{m}$ et $38 \mu \mathrm{m}$; la morphologie est typique des Megatrypanum, le kinétoplaste est petit, proche du noyau et éloigné de l'extrémité postérieure. Quelques individus sont striés de «myonèmes ». La taille du flagelle libre peut atteindre $10 \mu \mathrm{m}$.

b) «Formes géantes».

Elles n'ont été observées que sur les frottis de sang cardiaque et sur les appositions de poumon; leurs longueurs s'échelonnent entre 500 et $1400 \mu . \mathrm{m}$, et leurs largeurs entre 10 et $35 \mu \mathrm{m}$. Il est probable que cette grande taille les empêche de s'insinuer dans les capillaires, ce qui ne permettrait pas de les retrouver sur les frottis de sang périphérique. Comme tous les éléments volumineux, ils se rassemblent sur les bords $(P l . I: 1,3)$, et surtout à l'extrême queue du frottis (Pl. I : 2, 4). Les rares individus qui n'ont pas été entraînés sont les mieux préservés. Ces trypanosomes sont fragiles: souvent leur paroi se rompt à plusieurs niveaux, lors de la confection des frottis, et le cytoplasme se répand en petites flaques; les figures 2 et 4 de la planche II, ainsi que le schéma $H$ de la planche III, montrent ces zones endommagées. Le noyau est de petite taille, rond ou ovalaire $(5$ à $6 \mu \mathrm{m}$ de diamètre). Il est situé dans le second tiers de la longueur. Le kinétoplaste en bâtonnet, d'un demi- $\mu \mathrm{m}$ de long, est juxtanucléaire ; la flagelle, relativement court $(40 \mu \mathrm{m})$ par rapport à la taille du corps, est soit libre, soit porteur d'une petite membrane ondulante (Pl. III, A et $H)$. 

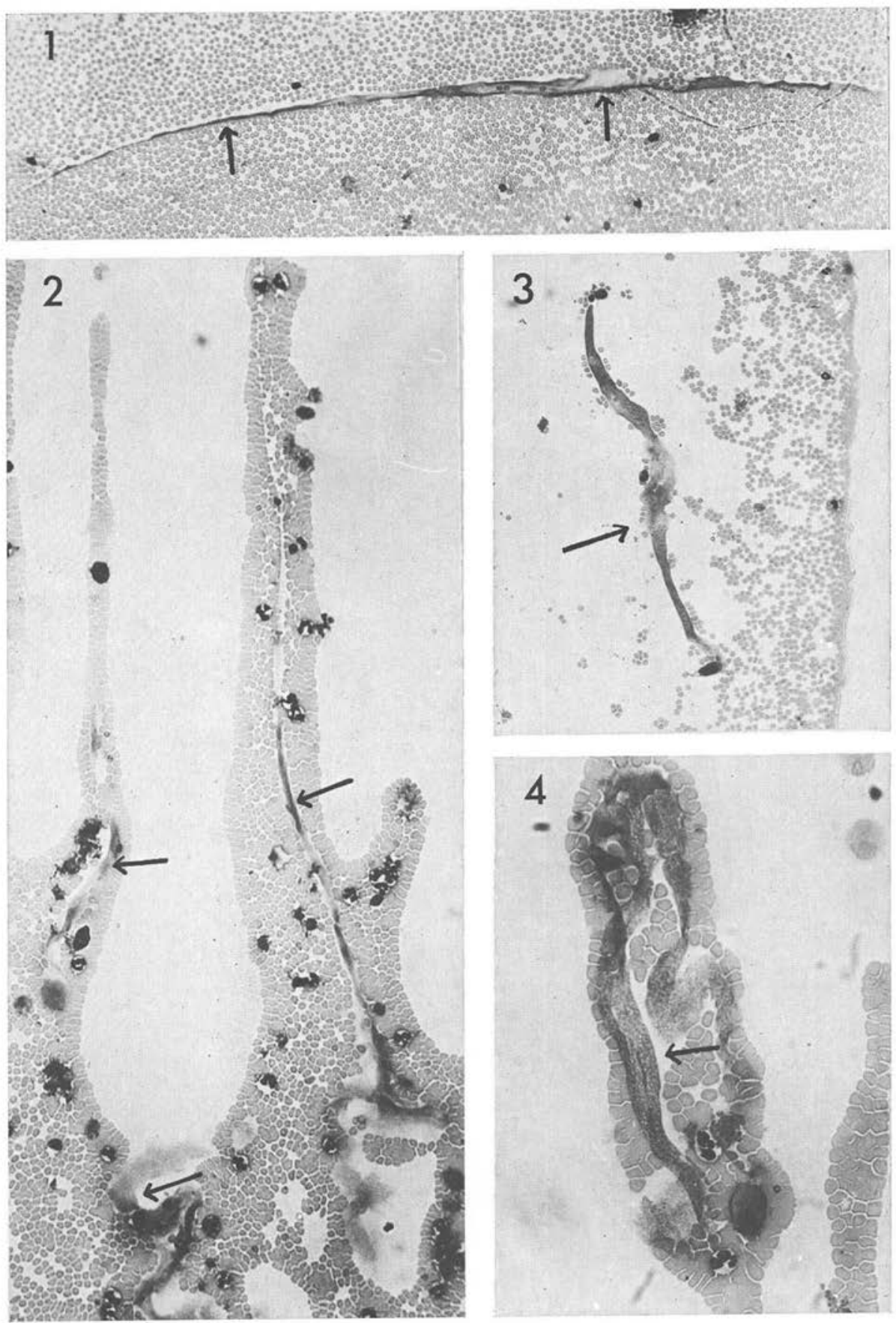

Planche I. 1 à 4. Photographies des différents aspects des formes géantes sur frottis sanguin. 

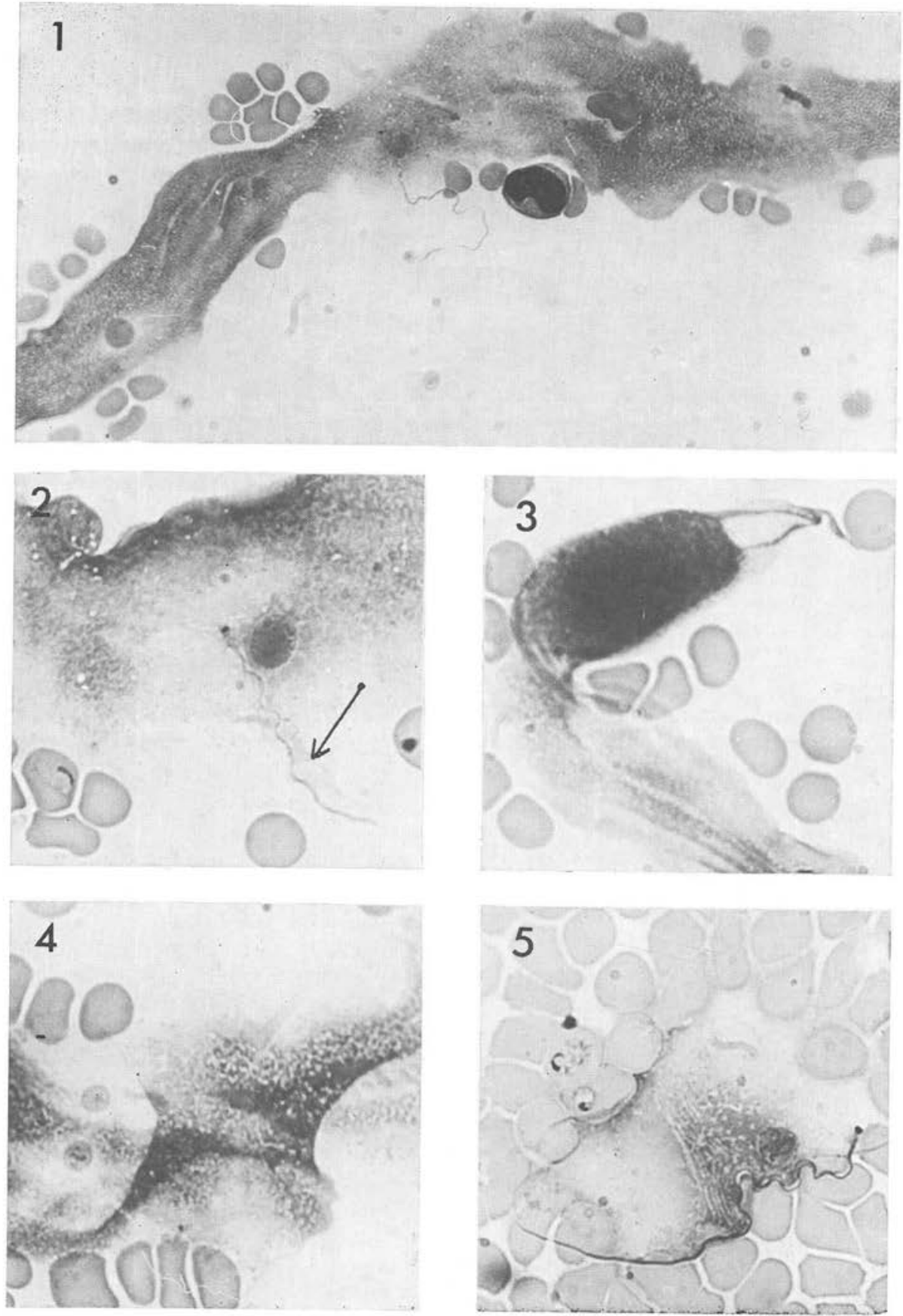

Planche II.

1 et 2. Détails des noyaux des kinétoplastes et des fiagelles des formes géantes. Sur la photographie $\mathrm{n}^{\circ} 2$, on distingue une membrane ondulante (flèche). 3. Extrémité d'une forme géante. 4. Image montrant la fragilité du corps des formes géantes, qui éclatent lors de la confection des frottis. 5. Aspect d'une forme de taille intermédiaire. 


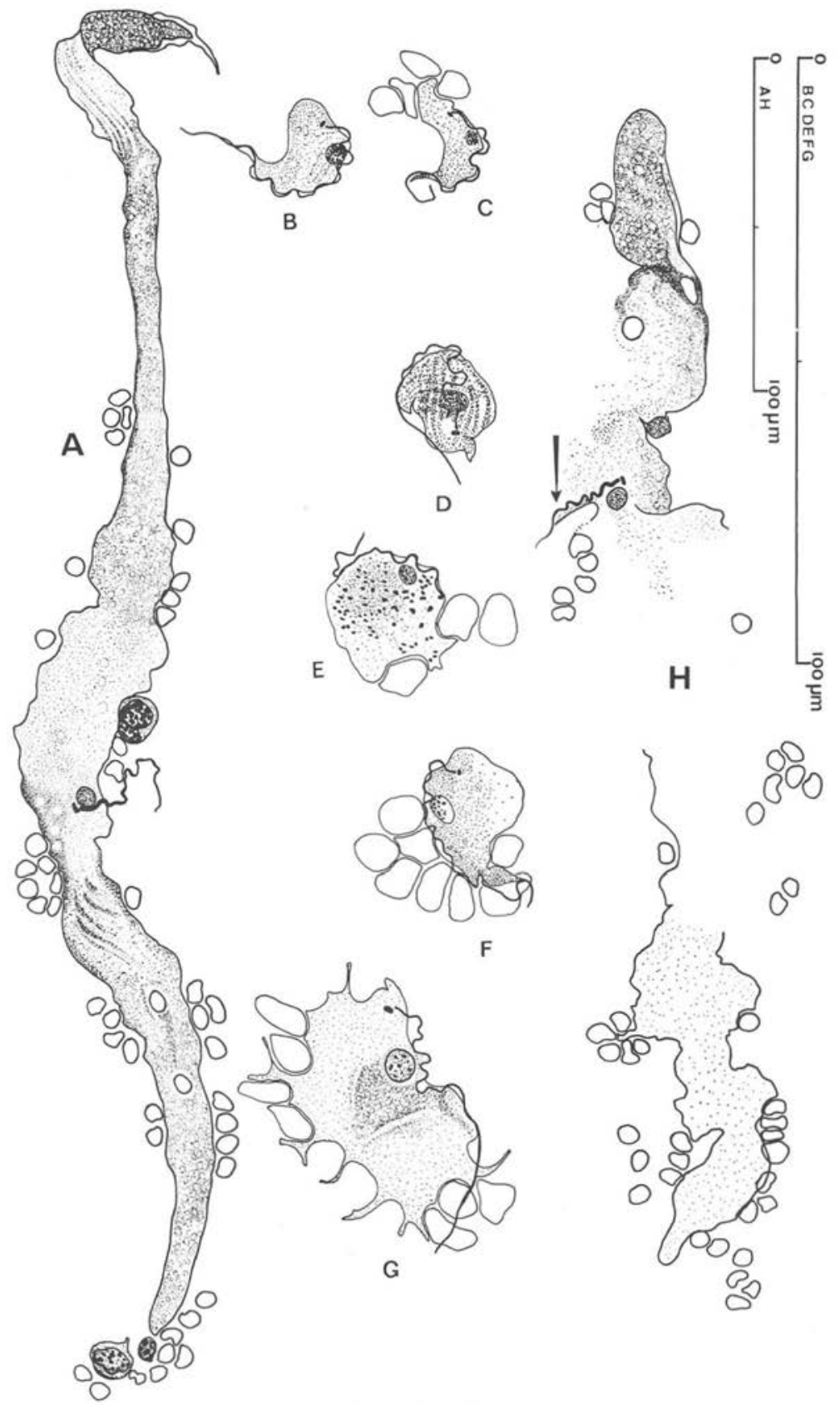

Planche III.

A et $\mathrm{H}$ : deux individus géants; le trypanosome A présente un flagelle libre: le trypanosome $\mathrm{H}$, dont la partie médiane a éclaté lors de la confection du frottis, présente un flagelle soulevant une membrane ondulante (flèche).

$\mathrm{B}$ et $\mathrm{C}$ : Petits trypomastigotes.

$D$ : Trypomastigote strié de myonèmes.

E : Sphaeromastigote.

F : Forme intermédiaire entre sphaeromastigote et trypomastigote.

$\mathrm{G}$ : Forme de taille intermédiaire. 
Dans certains cas, l'une des extrémités, dont nous ne pouvons savoir si elle est antérieure ou postérieure, car le flagelle, court, est latéro-médian, présente une formation très particulière $(P l . I, 4, P l . I I$, 3, et $P l$. III, A). C'est une masse ovale bien colorable par le Giemsa, plus dense que le reste du cytoplasme, qui se prolonge par un petit éperon peu ou pas coloré, à paroi épaisse; nous n'en connaissons pas la fonction.

c) Formes de taille intermédiaire.

Enfin, nous avons trouvé quelques formes de taille intermédiaire $(50 \mu \mathrm{m} \times 60 \mu \mathrm{m})$ $(P l . I I, 5$, et $I I I, D)$ dont le corps est strié de myonèmes, plus globuleuses, ayant un aspect plus classique de trypanosome que les formes gigantesques.

\section{Discussion}

Le problème qui se pose est de savoir si nous avons, chez ces Hipposideros cyclops, une ou plusieurs espèces de trypanosomes, l'une comprenant les formes gigantesques, l'autre les «petites formes » typiques du sous-genre Megatrypanum, ou si ces formes très dissemblables ne sont que les stades différents d'une même espèce. En faveur de cette dernière hypothèse, ne disposant que d'éléments morphologiques, nous retenons la grande variation de taille et d'aspect des «petites formes », ainsi que l'observation d'individus de taille intermédiaire ( $P l . I I I, G)$.

Aussi, nous avons décidé d'inclure tous ces trypanosomes d'Hipposideros cyclops dans une seule espèce, que nous rattachons au groupe Megadermae (Hoare, 1972) ; ce groupe, fort hétérogène, comprend tous les Megatrypanum de Chiroptères de l'Ancien et du Nouveau Monde; neuf espèces ont été individualisées, dont six en Afrique et trois en Amérique; de plus quelques trypanosomes africains ont été décrits en tant qu'espèces affines (heybergi-like et leleupi-like) (voir Marinkelle, 1976).

La morphologie générale des trois espèces américaines ( $T$. leonidasdeanei Zeledon et Rosabal, $T$. pessoai Deane et Sugay, et $T$. pifanoi Marinkelle et Duarte) est différente de celle des petites formes que nous avons observées chez Hipposideros cyclops. Ces espèces américaines sont moins trapues et ont toutes trois une extrémité postérieure très effilée; elles ont un aspect plus filiforme. Quant aux espèces africaines, $T$. lizae se distingue nettement de $T$. mpapuense Reichenow, ce dernier n'ayant pas de flagelle libre. $T$. lizae se distingue en outre des cinq autres espèces africaines (T. heybergi Rodhain, $T$. leleupi Rodhain, $T$. megadermae Wenyon, $T$. morinorum Léger et Baury, T. thomasi Lips et Rodhain) par la grande variabilité de taille des «petites formes» qui mesurent de 20 à $45 \mu \mathrm{m}$ (aucune des espèces africaines citées n'a une taille inférieure à $25 \mu \mathrm{m}$ ), et par leur largeur importante qui peut aller jusqu'à $30 \mu \mathrm{m}$.

Nous nommons cette nouvelle espèce Trypanosoma (Megatrypanum) lizae en hommage à Elizabeth U. Canning. 
Les lames types de ce matériel sont déposées au Muséum national d'Histoire naturelle sous les numéros P IV, 142 à 150 .

\section{Bibliographie}

Hoare C. A. (1964) : Morphological and taxonomic studies on mammalian trypanosomes. J. Protozool., 11, 200-207.

Hoare C. A. (1972) : «The Trypanosomes of Mammals, a zoological monograph ». Blackwell Scientific Publications, édit., Oxford. 749 p.

Landau I., Chabaud A. G. (1978) : Description de Plasmodium cyclopsi n. sp. parasite du Microchiroptère Hipposideros cyclops à Makokou (Gabon). Ann. Parasitol. Hum. Comp., 53, 247-253.

Marinkelle G. J. (1976) : In « Biology of the Kinetoplastida», Lumsden W. H. R. and Evans D. A. Edt. Academic Press, édit., Londres, 173-181. 